Oral Contraceptives and Myocardial Infarction

SiR,-Before the two most interesting studies by Dr. J. I. Mann and his colleagues (3 May, pp. 241 and 245) are accepted at face value there are a number of questions which need answering. It is notable, for instance, that in the first study only one out of the 60 cases of myocardial infarction had "current oral contraceptive use" as the sole "risk factor," and of the 50 oral contraceptive users in the second study, five $(10 \%)$ were under medical treatment for conditions whioh would lead to some other form of contraception being recommended at a family planning clinic. There are two possible explanations for this. Either these women were unsuitable for or would not accept alternative methods and the overriding need to prevent pregnancy made the oral contraceptive the lesser of two evils or the practitioners were exercising less care in their use of the oral contraceptive than is usual in family planning clinics. Pregnancy involves a greater hazard than does exposure to the oral contraceptive. Thus women with risk factors are more likely than those without to be using reliable methods of contraception. The proper control group should therefore be one representative of women using the intrauterine device or the cap, or referred for sterilization, rather than one representative of the general population. Until we know the comparable statistics for users of other methods the issue will remain in doubit.

A further cause of possible error in the second study is the fact that knowledge that the woman was taking the oral contraceptive may have been a factor influencing the doctor certifying the cause of death. No check was made on women centified as dying from other causes to ascertain if any of them might have had unrecognized infarots. What matters is whether the woman lives or dies, not what opinion as to cause of death appears on her certificate. The possibility that the oral contraceptive influences the mode or attribution of cause but not the fact of death should be excluded. There is a need for a well designed study comparing montality from all causes between users of the oral contracepive and users of other methods before pronouncements are made which may deter women from accepting the oral contraceptive.-I am, etc.,

Scunthorpe, Lincs

J. S. ROBERTSON

SIR, - I found the two articles by Dr. J. I. Mann and his colleagues on the association of myocardial infarction with oral contraceptives ( 3 May, pp. 241 and 245) very interesting but noticed that both omitted to mention selection of patients suitable for the administration of oral contraceptives.

Those of us who are trained by, and working for, the Family Planning Association know the importance of selection as regards raised blood pressure and obesity and that the regular checking of these is essential, but so many general practitioners simply hand out prescriptions on request with no sont of examination before or during administration. We also inquire about cigarette smoking, diabetes in the family, etc., and I feel that careful history-taking and check visits do something towards avoidance of arterial or venous thrombosis. -I am, etc.,

Hatch End, Middx

MAVIS STRATFORD

1 Davies, J. G., and Sowton, E., British Heart
Fournal, 1966, 28, 231.
2 Chatterjee, K., et al., Postgraduate Medical
Fournal, 1969, 45, 713.

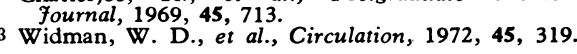

\section{Hormone Patterns in Anorexia Nervosa}

\section{Intracardiac Short Circuit}

SIR,-The article by Dr. I. J. Copperman and others (29 March, p. 722) calls for comment. The authors have presented no evidence to support their assumption of an intracardiac short circuit. Such a hypothesis is untenable, as a stimulus passing between electrodes would activate the myocardium unless the threshold for stimulation was too high for this to occur. In such a situation the ourrent could not be expected to pass selectively from one electrode to the other and be totally "wasted" between the distal end of the "free" electrode in the superior vena cava and the indifferent electrode of the pacemaker. The impedance of this pathway must approximate that between the tip of the other catheter and the pacemaker indifferent. Alternative explanations of the phenomenon observed are as follows.

(1) Loss of pacing in each instance was the result of poor contact with the right ventricular endocardium. Either the second electrode was placed in the coronary sinus on all three occasions or it was unstable in the right ventricular cavity. A rise in stimulation threshold leading to failure of capture after electrode positioning in the coronary sinus is well known. ${ }^{1}$ Coronary sinus positioning may be avoided by monitoring the electrogram from the catheter tip prior to measuring the stimulation threshold of a new electrode position. A typical picture of tall biphasic $\mathrm{P}$ waves in addition to all RS complexes identifies the coronary sinus. ${ }^{2}$

(2) The failure of pacing may have been due to false inhibition. Intermittent contact between two endocardial electrodes will generate electrical spikes (see fig.) of appropriate amplitude and frequency to inhibit demand pacemakers, and this has been observed clinically on several occasions. ${ }^{3}$ Less frequently a demand unit may be inhibited by the $P$ waves from an electrode in the coronary sinus.

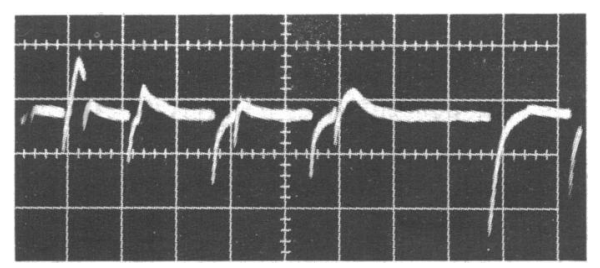
Polaroid photograph of signals generated in the laboratory between two platinum pacing electrodes in intermittent contact in blood at body temperature. Calibrations:
$0.5 \mathrm{~s} /$ divisions.

We would conclude that the problem might have been avoided by a more careful pacing technique; in particular, the monitoring of the electrogram from the catheter electrode tip during positioning would have revealed the true situation. We consider the recommendation of epicardial pacing to be unnecessary.-We are, etc.,

RICHARD SUTTON LYN BRIERS

National Heart Hospital,
London W.1 JOHN NORMAN
SIR,-Your leading anticle on this subject (12 April, p. 52) contains the seriously misleading statement that "in normal pubertal and adolescent girls the weight at which the menarche occurs has been identified as $47 \cdot 8 \pm 0.5 \mathrm{~kg}$." It is perhaps important for a professional auxologist to assure your readers that this statement is indeed the nonsense that common sense and observation would lead one to believe. Girls reach menarche at a wide range of weights; in a recent paper Johnston et al. ${ }^{1}$ quote a range of 25.9 to $97.3 \mathrm{~kg}$ in eight studies of normal girls.

You have failed to understand the original work of Frisch and her colleagues, in which in certain samples it was shown that the mean weight of groups of girls who matured relatively early was not significantly different from the mean weight at menarche of those critical discussion of these findings has made clear that even this relationship does not hold in all groups of normal girls. The idea an individual can be "identified," like a steroid in gas-liquid chromatographic analys:s, is quite false.-I am, etc.,

Institute of Child Health,

J. M. TANNER Johnston, F. E., et al., American fournal of
Diseases of Children, 1975, 129, 19.

** Professor Tanner is correct in drawing attention to the scatter of menarchal weights. Frisch and her colleagues ${ }^{1}$ in fact made the same observation, though the scatter they observed was much less $(32-64 \mathrm{~kg})$. However, they also claimed in their "critical weight" hypothesis that it is weight-related events that determine menarche irrespective of age. This weight band was identified by them as $47.8 \pm 4.6 \mathrm{~kg}$ (the $25 \mathrm{th}$ percentile of weight at menarche in their population was $43 \mathrm{~kg}$ and the $75 \mathrm{th}$ was $51.5 \mathrm{~kg}$ ). The figure of $47.8 \pm 0.5 \mathrm{~kg}$ referred to this mean menarchal weight together with the standard error, not the standard deviation as might have been inferred from the leading article, though, as Professor Tanner suggests, common sense requires the former interpretation. Frisch has, of course, subsequently become more interested in fatness as a determinant of menarche. She claims that for the majority of females per cent. body fatness is an even more critical determinant of menarche than weight. Its variability at menarche is over $50 \%$ less than that of weight and it is an even more stable indicant adolescent growth in relation to her peers. ${ }^{2}$ However, she still acknowledges the existence of deviant subgroups in respect of both weight and per cent. body fatness and their relation to menarche. The point of the leading article was more to draw attention to (1) the very critical weight threshold seeming to govern pituitary responsivity to $50 \mu \mathrm{g}$ L.H./F.S.H./R.H. in patients with anorexia nervosa and (2) the probably related clinical relevance for anorexia nervosa of what is, in who matured relatively late. Subsequent that a weight at which menarche occurs in of the individual's pattern of pubertal and 